\title{
Deuteron electromagnetic structure functions and polarization properties in soft-wall AdS/QCD
}

\author{
Thomas Gutsche, ${ }^{1}$ Valery E. Lyubovitskij, ${ }^{1,2,3}$ and Ivan Schmidt $^{4}$ \\ ${ }^{1}$ Institut für Theoretische Physik, Universität Tübingen, \\ Kepler Center for Astro and Particle Physics, Auf der Morgenstelle 14, D-72076 Tübingen, Germany \\ ${ }^{2}$ Department of Physics, Tomsk State University, 634050 Tomsk, Russia \\ ${ }^{3}$ Laboratory of Particle Physics, Mathematical Physics Department, \\ Tomsk Polytechnic University, Lenin Avenue 30, 634050 Tomsk, Russia \\ ${ }^{4}$ Departamento de Física y Centro Científico Tecnológico de Valparaíso (CCTVal), \\ Universidad Técnica Federico Santa María, Casilla 110-V, Valparaíso, Chile
}

(Dated: June 22, 2021)

\begin{abstract}
We apply a soft-wall AdS/QCD approach to the description of deuteron structure functions and tensor polarized properties. Present work is a completion of our previous study on electromagnetic form factors. By the appropriate choice of two couplings in the effective action we are able to reproduce both the form factors and structure functions in full consistency with model-independent constraints set by perturbative QCD. Our framework is based on a five-dimensional action in AdS space formulated in terms of vector fields dual to the deuteron and the electromagnetic fields. The AdS fields depend on four Minkowski and one holographic coordinate $z$. The scale dependence of the form factors and structure functions is consistent with quark counting rules implying the $1 / Q^{10}$ behavior of the charge form factor $G_{C}\left(Q^{2}\right)$, structure functions $A\left(Q^{2}\right)$ and $B\left(Q^{2}\right)$, and the $1 / Q^{12}$ behavior of the magnetic $G_{M}\left(Q^{2}\right)$ and quadrupole $G_{Q}\left(Q^{2}\right)$ form factors.
\end{abstract}

PACS numbers: 11.10.Kk,11.25.Tq,12.38.Lg,13.40.Gp

Keywords: deuteron, electromagnetic form factors and structure functions, gauge-gravity duality, AdS/QCD

\section{INTRODUCTION}

The study of electron-deuteron scattering gives a unique insight into the structure of the deuteron. The structure information is contained in the gauge-invariant matrix element describing the interaction of the deuteron with the electromagnetic field, which reads as

$$
\begin{aligned}
M_{\mathrm{inv}}^{\mu}\left(p, p^{\prime}\right) & =-\left(G_{1}\left(Q^{2}\right) \epsilon^{*}\left(p^{\prime}\right) \cdot \epsilon(p)-\frac{G_{3}\left(Q^{2}\right)}{2 M_{d}^{2}} \epsilon^{*}\left(p^{\prime}\right) \cdot q \epsilon(p) \cdot q\right)\left(p+p^{\prime}\right)^{\mu} \\
& -G_{2}\left(Q^{2}\right)\left(\epsilon^{\mu}(p) \epsilon^{*}\left(p^{\prime}\right) \cdot q-\epsilon^{* \mu}\left(p^{\prime}\right) \epsilon(p) \cdot q\right)
\end{aligned}
$$

where $\epsilon\left(\epsilon^{*}\right)$ and $p\left(p^{\prime}\right)$ are the polarization and four-momentum of the initial (final) deuteron, $q=p^{\prime}-p$ is the momentum transfer and $M_{d}$ is the deuteron mass.

The three electromagnetic (EM) form factors $G_{1,2,3}$ of the deuteron are related to the charge $G_{C}$, quadrupole $G_{Q}$ and magnetic $G_{M}$ form factors by

$$
G_{C}=G_{1}+\frac{2}{3} \tau_{d} G_{Q}, \quad G_{M}=G_{2}, \quad G_{Q}=G_{1}-G_{2}+\left(1+\tau_{d}\right) G_{3}, \quad \tau_{d}=\frac{Q^{2}}{4 M_{d}^{2}} .
$$

The form factors are normalized at $Q^{2}=0$ as

$$
G_{C}(0)=1, \quad G_{Q}(0)=M_{d}^{2} \mathcal{Q}_{d}=25.83, \quad G_{M}(0)=\frac{M_{d}}{M_{N}} \mu_{d}=1.714,
$$

where $M_{N}$ is the nucleon mass, $\mathcal{Q}_{d}=7.3424 \mathrm{GeV}^{-2}$ and $\mu_{d}=0.8574$ are the quadrupole and magnetic moments of the deuteron. 
The set of the three form factors $\left(G_{C}\left(Q^{2}\right), G_{M}\left(Q^{2}\right), G_{Q}\left(Q^{2}\right)\right)$ and their combinations - structure functions

$$
A\left(Q^{2}\right)=G_{C}^{2}\left(Q^{2}\right)+\frac{2}{3} \tau_{d} G_{M}^{2}\left(Q^{2}\right)+\frac{8}{9} \tau_{d}^{2} G_{Q}^{2}\left(Q^{2}\right), \quad B\left(Q^{2}\right)=\frac{4}{3} \tau_{d}\left(1+\tau_{d}\right) G_{M}^{2}\left(Q^{2}\right) .
$$

are the main observables defining the electromagnetic structure of the deuteron (for a recent review on the experimental and theoretical progress see Ref. [1-4]). In Ref. [5-7] model-independent relations between the deuteron form factors and their individual scalings imposed by perturbative QCD at large Euclidean values of $Q^{2}$ have been derived. In particular, in Ref. [5] it was shown that $\sqrt{A\left(Q^{2}\right)}$ can be factorized in terms of the nucleon form factor $F_{N}\left(Q^{2} / 4\right)$ and the so-called "reduced" nuclear form factor $f_{d}\left(Q^{2}\right)$ as

$$
\sqrt{A\left(Q^{2}\right)}=f_{d}\left(Q^{2}\right) F_{N}^{2}\left(Q^{2} / 4\right) .
$$

$\sqrt{A\left(Q^{2}\right)}$ scales as $1 / Q^{10}$ in agreement with quark counting rules, since the deuteron has six constituent quarks. Ref. [6] contains a derivation of the high $Q^{2} \mathrm{QCD}$ prediction for the following relation between the charge and quadrupole form factors

$$
G_{C}\left(Q^{2}\right)=\frac{2}{3} \tau_{d} G_{Q}\left(Q^{2}\right) .
$$

In Ref. 7] this relation has been extended by including the next-to-leading term in the form

$$
G_{C}\left(Q^{2}\right)=\left(\frac{2}{3} \tau_{d}-1\right) G_{Q}\left(Q^{2}\right) .
$$

In addition it was shown that the three deuteron form factors and structure functions scale at large $Q^{2}$ as

$$
\begin{aligned}
& G_{C}\left(Q^{2}\right): G_{M}\left(Q^{2}\right): G_{Q}\left(Q^{2}\right)=\left(\frac{2}{3} \tau_{d}-1\right): 2:-1, \\
& B\left(Q^{2}\right): A\left(Q^{2}\right): G_{C}^{2}\left(Q^{2}\right)=4: 1: \frac{1}{3} .
\end{aligned}
$$

Using these results of Refs. [5 7] one concludes that the individual deuteron form factors and structure functions scale at large $Q^{2}$ as

$$
\begin{aligned}
\sqrt{A\left(Q^{2}\right)} & \sim \sqrt{B\left(Q^{2}\right)} \sim G_{C}\left(Q^{2}\right) \sim \frac{1}{Q^{10}}, \\
G_{M}\left(Q^{2}\right) & \sim G_{Q}\left(Q^{2}\right) \sim \frac{1}{Q^{12}} .
\end{aligned}
$$

The cross section for elastic electron-deuteron scattering is defined by the Rosenbluth formula

$$
\begin{aligned}
\frac{d \sigma}{d \Omega} & =\sigma_{M} S\left(Q^{2}\right), \\
S\left(Q^{2}\right) & =A\left(Q^{2}\right)+B\left(Q^{2}\right) \tan ^{2} \frac{\theta}{2},
\end{aligned}
$$

where $\sigma_{M}$ is the Mott cross section and $\theta$ is the electron scattering angle.

Other important characteristics of the deuteron are the deuteron tensor-polarized observables (tensor analyzing powers) $T_{20}, T_{21}$ and $T_{22}$, which are expressed in terms of the three form factors $\left(G_{C}\left(Q^{2}\right), G_{M}\left(Q^{2}\right), G_{Q}\left(Q^{2}\right)\right)$ and scattering angle $\theta$ as

$$
\begin{aligned}
T_{20}\left(Q^{2}, \theta\right) & =-\frac{1}{S\left(Q^{2}\right) \sqrt{2}}\left[\frac{8}{3} \tau_{d} G_{C}\left(Q^{2}\right) G_{Q}\left(Q^{2}\right)\right. \\
& \left.+\frac{8}{9} \tau_{d}^{2} G_{Q}^{2}\left(Q^{2}\right)+\frac{\tau_{d}}{3}\left(1+2\left(1+\tau_{d}\right) \tan ^{2} \frac{\theta}{2}\right) G_{M}^{2}\left(Q^{2}\right)\right], \\
T_{21}\left(Q^{2}, \theta\right) & =-\frac{2}{S\left(Q^{2}\right) \sqrt{3}} \sqrt{\tau_{d}^{3}\left(1+\tau_{d} \sin ^{2} \frac{\theta}{2}\right)} \frac{G_{M}\left(Q^{2}\right) G_{Q}\left(Q^{2}\right)}{\cos \frac{\theta}{2}}, \\
T_{22}\left(Q^{2}, \theta\right) & =-\frac{1}{2 S\left(Q^{2}\right) \sqrt{3}} \tau_{d} G_{M}^{2}\left(Q^{2}\right) .
\end{aligned}
$$


As shown in Ref. [15] it is useful to define the quantity

$$
\tilde{T}_{20}\left(Q^{2}\right)=\frac{1}{1-\delta}\left(T_{20}\left(Q^{2}\right)+\frac{\delta}{2 \sqrt{2}}\right)
$$

with the use of the factor

$$
\delta=\frac{B\left(Q^{2}\right)}{S\left(Q^{2}\right)}\left[\frac{1}{2\left(1+\tau_{d}\right)}+\tan ^{2} \frac{\theta}{2}\right]
$$

in order to eliminate the dependence of $T_{20}$ on $G_{M}$ and $\theta$ :

$$
\tilde{T}_{20}\left(Q^{2}\right)=-\frac{\tau_{d}}{\sqrt{2}} \frac{3 \beta\left(Q^{2}\right)+\tau_{d}}{\frac{9}{8} \beta^{2}\left(Q^{2}\right)+\tau_{d}^{2}},
$$

where $\beta\left(Q^{2}\right)=G_{C}\left(Q^{2}\right) / G_{Q}\left(Q^{2}\right)$ is the ratio of $G_{C}$ and $G_{Q}$ form factors. By further elimination of the leading $Q^{2}$ dependence one can introduce the reduced quantity

$$
\begin{aligned}
\tilde{T}_{20 R}\left(Q^{2}\right) & =-\frac{3}{Q^{2} Q_{d} \sqrt{2}} \tilde{T}_{20}\left(Q^{2}\right) \\
& =\frac{G_{Q}\left(Q^{2}\right)}{G_{Q}(0)} \frac{G_{C}\left(Q^{2}\right)+\frac{\tau_{d}}{3} G_{Q}\left(Q^{2}\right)}{G_{C}^{2}\left(Q^{2}\right)+\frac{8}{9} \tau_{d}^{2} G_{Q}^{2}\left(Q^{2}\right)}
\end{aligned}
$$

which is normalized as $\tilde{T}_{20 R}(0)=1$ for $Q^{2}=0$.

Using the power scalings of the deuteron form factors (9) one can derive large $Q^{2}$ scalings for the tensor-polarized observables [7] as

$$
\begin{aligned}
& T_{20}\left(Q^{2}\right) \sim-\sqrt{2} \frac{1+\tan ^{2} \frac{\theta}{2}}{1+4 \tan ^{2} \frac{\theta}{2}} \sim \mathcal{O}(1), \\
& T_{21}\left(Q^{2}\right) \sim \sqrt{3} \frac{\tan \frac{\theta}{2}}{1+4 \tan ^{2} \frac{\theta}{2}} \sim \mathcal{O}(1), \\
& T_{22}\left(Q^{2}\right) \sim-\frac{\sqrt{3}}{2 \tau_{d}} \frac{1}{1+4 \tan ^{2} \frac{\theta}{2}} \sim \mathcal{O}\left(1 / Q^{2}\right), \\
& \tilde{T}_{20}\left(Q^{2}\right) \sim-\sqrt{2} \sim \mathcal{O}(1), \\
& \tilde{T}_{20 R}\left(Q^{2}\right) \sim \frac{3}{4 \tau_{d} G_{Q}(0)} \sim \mathcal{O}\left(1 / Q^{2}\right) .
\end{aligned}
$$

In the present paper we further improve the calculation on the deuteron form factors in a soft-wall AdS/QCD approach [8 11], started in Ref. [4]. We present in addition the results for the structure functions and the tensorpolarized variables. Our approach is based on gauge/gravity duality and is constructed as a holographic dual to pQCD. The main advantage of our framework is that it gives a description of the deuteron electromagnetic form factors in the full $Q^{2}$ regime and guarantees the correct power scaling of form factors and structure functions at large $Q^{2}$. Note that this important property of approaches based on gauge/string duality originally was originally found in Ref. [12] and was later confirmed in Refs. [4, 9 11].

\section{FORMALISM AND NUMERICAL RESULTS}

The formalism is based on an effective action given in terms of the AdS fields $d^{M}(x, z)$ and $V^{M}(x, z)$. These fields are dual to the Fock component contributing to the deuteron with twist $\tau=6$ and the electromagnetic field, respectively. The action is given by

$$
\begin{aligned}
S & =\int d^{4} x d z \sqrt{g} e^{-\varphi(z)}\left[-\frac{1}{4} F_{M N} F^{M N}-D^{M} d_{N}^{\dagger} D_{M} d^{N}-i c_{2}(z) F^{M N} d_{M}^{\dagger} d_{N}\right. \\
& \left.+\frac{c_{3}(z)}{4 M_{d}^{2}} e^{2 A(z)} \partial^{M} F^{N K}\left(i D_{K} d_{M}^{\dagger} d_{N}-d_{M}^{\dagger} i D_{K} d_{N}+\text { H.c. }\right)+d_{M}^{\dagger}\left(\mu^{2}+U(z)\right) d^{M}\right],
\end{aligned}
$$


where $g=\left|\operatorname{detg}_{\mathrm{MN}}\right|=e^{10 A(z)}$ and $A(z)=\log (R / z) ; F^{M N}$ is the stress tensor of vector field $V^{M} ; D^{M}$ is the covariant derivative including $V^{M} ; \mu^{2} R^{2}=(\Delta-1)(\Delta-3)$ is the five-dimensional mass; $R$ is the $\operatorname{AdS} \operatorname{radius,} \varphi(z)=\kappa^{2} z^{2}$ is the background dilaton field and $\kappa$ is the scale parameter; $\Delta=\tau+1$ is the dimension of the $d^{M}(x, z)$ field; $M_{d}$ is the deuteron mass and $U(z)=U_{0} \varphi(z) / R^{2}$ is the confinement potential, where the constant $U_{0}$ is fixed by the value of the deuteron mass. We work in the axial gauge for both vector fields $d^{z}(x, z)=0$ and $V^{z}(x, z)=0$. The $z$-dependent couplings $c_{2}(z)$ and $c_{3}(z)$ are constrained by the normalization and the large $Q^{2}$ scaling of the deuteron electromagnetic form factors (see discussion below).

This updated action is an extension of the deuteron action suggested in Ref. 4]. The main difference is that now we incorporate the $z$-dependence of the AdS/QCD couplings $c_{2}$ and $c_{3}$ :

$$
\begin{aligned}
& c_{2}(z)=e^{-\beta \varphi(z)}\left[c_{2}^{(1)}+c_{2}^{(2)} e^{\alpha_{2} \log \varphi(z)}\right], \\
& c_{3}(z)=c_{3} e^{-\beta \varphi(z)+\alpha_{3} \log \varphi(z)},
\end{aligned}
$$

where the couplings $c_{2}^{(1)}, c_{2}^{(2)}$ and $c_{3}$ are fixed from the normalization of $G_{M}\left(Q^{2}\right), G_{Q}\left(Q^{2}\right)$ and the asymptotics of $G_{M}\left(Q^{2}\right)$ at large $Q^{2}$ :

$$
\begin{aligned}
c_{2}^{(1)} & =12 \int_{0}^{1} d x \frac{(1-x)^{5}}{[1+\beta(1-x)]^{7}}, \\
c_{2}^{(2)} & =G_{2}(0)-c_{2}^{(1)}=\frac{M_{d}}{M_{N}} \mu_{d}-c_{2}^{(1)}, \\
c_{3} & =G_{3}(0)=M_{d}^{2} Q_{d}-1+\frac{M_{d}}{M_{N}} \mu_{d} .
\end{aligned}
$$

The parameters $\alpha_{2}, \alpha_{3}$ and $\beta$ should satisfy the conditions

$$
\alpha_{2}>0, \quad \alpha_{3}>1, \quad \beta \geq 0
$$

to guarantee the scaling behavior (9) of the deuteron form factors and structure function. Their numerical values are optimized in a best fit to data:

$$
\alpha_{2}=0.25, \alpha_{3}=1.1, \beta=1.2
$$

Our calculation of deuteron form factors proceeds in several steps. First, we perform a Kaluza-Klein (KK) decomposition of the vector AdS field dual to the deuteron $d^{\mu}(x, z)=\exp [(\varphi(z)-A(z)) / 2] \sum_{n} d_{n}^{\mu}(x) \Phi_{n}(z)$, where $d_{n}^{\mu}(x)$ is the tower of KK fields dual to the deuteron fields with radial quantum number $n$ and twist-dimension $\tau=6$, and $\Phi_{n}(z)$ are their bulk profiles in the fifth direction of the AdS space.

Second, we derive a Schrödinger-type equation of motion (EOM) for the profile $\Phi_{n}(z)$

$$
\left[-\frac{d^{2}}{d z^{2}}+\frac{99}{4 z^{2}}+\kappa^{4} z^{2}+\kappa^{2} U_{0}\right] \Phi_{n}(z)=M_{d, n}^{2} \Phi_{n}(z),
$$

which is solved analytically

$$
\Phi_{n}(z)=\sqrt{\frac{2 n !}{(n+5) !}} \kappa^{6} z^{11 / 2} e^{-\kappa^{2} z^{2} / 2} L_{n}^{5}\left(\kappa^{2} z^{2}\right), \quad M_{d, n}^{2}=4 \kappa^{2}\left[n+3+\frac{U_{0}}{4}\right],
$$

where $L_{n}^{m}(x)$ are the generalized Laguerre polynomials. Restricting our considerations to the ground state $n=0$ we get $M_{d}=2 \kappa \sqrt{3+\frac{U_{0}}{4}}$. Using the central experimental value for the deuteron mass $M_{d}=1.875613 \mathrm{GeV}$ and setting the parameter $\kappa=190 \mathrm{MeV}$ (obtained from a fit to the data of the electromagnetic deuteron form factors), we fix $U_{0}=85.4494$. Note that the deuteron scale parameter is two times smaller than the analogous parameter $\kappa_{N} \simeq 380 \mathrm{MeV}$ entering in the description of the nucleon - mass and electromagnetic form factors [10, 11]. The difference between the nucleon and deuteron scale parameters can be related to the change of size of the hadronic systems - the deuteron as a two-nucleon bound state is about 2 times larger than the nucleon.

Third, we perform a Fourier transformation of the vector field $V(x, z)$ with respect to the Minkowski coordinate

$$
V_{\mu}(x, z)=\int \frac{d^{4} q}{(2 \pi)^{4}} e^{-i q x} V_{\mu}(q) V(q, z)
$$


and derive an EOM for the vector bulk-to-boundary propagator $V(q, z)$ dual to the $q^{2}$-dependent electromagnetic current

$$
\partial_{z}\left(\frac{e^{-\varphi(z)}}{z} \partial_{z} V(q, z)\right)+q^{2} \frac{e^{-\varphi(z)}}{z} V(q, z)=0
$$

The solution of this equation in terms of the gamma $\Gamma(n)$ and Tricomi $U(a, b, z)$ functions reads

$$
V(q, z)=\Gamma\left(1-\frac{q^{2}}{4 \kappa^{2}}\right) U\left(-\frac{q^{2}}{4 \kappa^{2}}, 0, \kappa^{2} z^{2}\right) .
$$

In the Euclidean region it is convenient to use the integral representation for $V(Q, z)[14]$

$$
V(Q, z)=\kappa^{2} z^{2} \int_{0}^{1} \frac{d x}{(1-x)^{2}} x^{a} e^{-\kappa^{2} z^{2} \frac{x}{1-x}},
$$

where $x$ is the light-cone momentum fraction and $a=Q^{2} /\left(4 \kappa^{2}\right)$.

With the present set-up the deuteron form factors can be easily calculated as:

$$
\begin{aligned}
G_{1}\left(Q^{2}\right) & =\int_{0}^{\infty} d z \Phi_{0}^{2}(z) V(Q, z), \\
G_{i}\left(Q^{2}\right) & =\int_{0}^{\infty} d z c_{i}(z) \Phi_{0}^{2}(z) V(Q, z), \quad i=2,3 .
\end{aligned}
$$

For a detailed discussion on the calculational technique see for example Ref. [4]. The explicit expressions for the form factors are

$$
\begin{aligned}
G_{1}\left(Q^{2}\right) & =\frac{\Gamma(a+1) \Gamma(7)}{\Gamma(a+7)}, \\
G_{2}\left(Q^{2}\right) & =c_{2}^{(1)} \frac{I_{1}\left(Q^{2}\right)}{I_{1}(0)}+c_{2}^{(2)} \frac{I_{2}\left(Q^{2}\right)}{I_{2}(0)} \\
G_{3}\left(Q^{2}\right) & =c_{3} \frac{I_{3}\left(Q^{2}\right)}{I_{3}(0)}
\end{aligned}
$$

where

$$
\begin{aligned}
& I_{i}\left(Q^{2}\right)=\frac{\Gamma\left(7+\Delta_{i}\right)}{\Gamma(6)} \int_{0}^{1} d x x^{a} \frac{(1-x)^{5+\Delta_{i}}}{(1+\beta(1-x))^{7+\Delta_{i}}}, \\
& \Delta_{1} \equiv 0, \quad \Delta_{2}=\alpha_{2}, \quad \Delta_{3}=\alpha_{3}, \quad c_{2}^{(1)}=2 I_{1}(0) .
\end{aligned}
$$

Note that the scaling of our form factors, the structure functions and tensor-polarized observables is fully consistent with perturbative QCD [5-7]. In particular, the structure functions $A\left(Q^{2}\right), B\left(Q^{2}\right)$ and the charge form factor $G_{C}\left(Q^{2}\right)$ have the correct power-scaling $1 / Q^{10}$ at large $Q^{2} \rightarrow \infty$, while the magnetic and quadrupole form factors scale as $1 / Q^{12}$ at large $Q^{2}$. Our results for the charge $G_{C}\left(Q^{2}\right)$, quadrupole $G_{Q}\left(Q^{2}\right)$ and magnetic $G_{M}\left(Q^{2}\right)$ form factors, structure functions $A\left(Q^{2}\right)$ and $B\left(Q^{2}\right)$, tensor-polarized quantities $T_{20}\left(Q^{2}\right), \tilde{T}_{20 R}\left(Q^{2}\right), T_{21}\left(Q^{2}\right)$ and $T_{22}\left(Q^{2}\right)$ are shown in Fig. 13. Results are compared to data taken from Refs. [1, 13] and references within. The shaded bands correspond to values for the scale parameter $\kappa$ in the range of $150-250 \mathrm{MeV}$. An increase of the parameter $\kappa$ leads to an enhancement of the form factors. The best description of the data on the deuteron form factors is obtained for $\kappa=190 \mathrm{MeV}$ and is shown by the solid line. We also determine the deuteron charge $r_{C}=\left(-6 d G_{C}\left(Q^{2}\right) /\left.d Q^{2}\right|_{Q^{2}=0}\right)^{1 / 2}$ and magnetic $r_{M}=\left(-6 d G_{M}\left(Q^{2}\right) /\left.d Q^{2}\right|_{Q^{2}=0} / G_{M}(0)\right)^{1 / 2}$ radii, which for $\kappa=190 \mathrm{MeV}$ are equal to $1.92 \mathrm{fm}$ and $2.26 \mathrm{fm}$, respectively. These values compare well with the data of $r_{C}=2.13 \pm 0.01 \mathrm{fm}$ and $r_{M}=1.90 \pm 0.14 \mathrm{fm}$.

In the soft-wall AdS/QCD approach we are able to describe of full $Q^{2}$ behavior of the electromagnetic structure of the deuteron including the tensor-polarized observables. One exception is the tensor analyzing power $T_{20}$ where discrepancies between prediction and data occur for $Q$ larger than $4 \mathrm{fm}^{-1}$. This mismatch can be traced to the 
behavior of $G_{C}\left(Q^{2}\right)$, where the prediction overestimates the data for large Q. The predicted behavior of $G_{C}\left(Q^{2}\right)$ can further be linked to the electromagnetic form factor $G_{1}\left(Q^{2}\right)$ contributing in the form

$$
G_{C}=G_{1}+\frac{2}{3} \tau_{d} G_{Q}
$$

While the prediction for $G_{1}\left(Q^{2}\right)$ is positive for all values of $Q^{2}$, the corresponding data of $G_{C}$ require a $G_{1}$ which should cross zero around $Q \sim 4 \mathrm{fm}^{-1}$. As an illustration in Fig. 4 we present two plots for $G_{C}$ - the exact result (left panel) and where the form factor $G_{1}$ is dropped (right panel), i.e. $G_{C} \simeq \tilde{G}_{C}=\frac{2}{3} \tau_{d} G_{Q}$ with a $\kappa$ of $190 \mathrm{MeV}$ and a variation in $\beta$ from 0 to 2 . Similar plots for the magnetic, quadrupole form factors and the structure functions are shown in Figs. 5 and 6. In all plots an increasing $\beta$ value leads to increase of all observables in the $Q^{2}$ dependence.

From Fig. 4 it is apparent that the form factor $G_{1}\left(Q^{2}\right)$ should cross zero around $Q \simeq 4 \mathrm{fm}^{-1}$ in order to suppress the contribution of the $G_{Q}$ form factor in $G_{C}$.

Finally, in Figs. 7-9 we show the sensitivity of all form factors and structure functions on the variation of the parameters $\alpha_{2}$ and $\alpha_{3}: 0 \leq \alpha_{2} \leq 1,1 \leq \alpha_{3} \leq 2$.

\section{Acknowledgments}

This work was supported by the German Bundesministerium für Bildung und Forschung (BMBF) under Project 05P2015 - ALICE at High Rate (BMBF-FSP 202): "Jet- and fragmentation processes at ALICE and the parton structure of nuclei and structure of heavy hadrons", by CONICYT (Chile) Research Project No. 80140097, by FONDECYT (Chile) under Grants No. 1140390 and FB - 0821, by Tomsk State University Competitiveness Improvement Program and the Russian Federation program "Nauka" (Contract No. 0.1526.2015, 3854).

[1] R. J. Holt and R. Gilman, Rep. Prog. Phys. 75, 086301 (2012).

[2] L. E. Marcucci et al., J. Phys. G: Nucl. Part. Phys. 43, 023002 (2016).

[3] Y. B. Dong, A. Faessler, T. Gutsche, and V. E. Lyubovitskij, Phys. Rev. C 78, 035205 (2008).

[4] T. Gutsche, V. E. Lyubovitskij, I. Schmidt and A. Vega, Phys. Rev. D 91, 114001 (2015).

[5] S. J. Brodsky, C. R. Ji, and G. P. Lepage, Phys. Rev. Lett. 51, 83 (1983).

[6] C. E. Carlson and F. Gross, Phys. Rev. Lett. 53, 127 (1984).

[7] S. J. Brodsky and J. R. Hiller, Phys. Rev. D 46, 2141 (1992).

[8] A. Karch, E. Katz, D. T. Son, and M. A. Stephanov, Phys. Rev. D 74, 015005 (2006); O. Andreev, Phys. Rev. D 73, 107901 (2006).

[9] S. J. Brodsky and G. F. de Teramond, Phys. Rev. D 77, 056007 (2008).

[10] T. Gutsche, V. E. Lyubovitskij, I. Schmidt, and A. Vega, Phys. Rev. D 85, 076003 (2012).

[11] T. Gutsche, V. E. Lyubovitskij, I. Schmidt, and A. Vega, Phys. Rev. D 86, 036007 (2012); Phys. Rev. D 87, 016017 (2013); Phys. Rev. D 87, 056001 (2013); A. Vega, I. Schmidt, T. Branz, T. Gutsche, and V. E. Lyubovitskij, Phys. Rev. D 80, 055014 (2009); T. Branz, T. Gutsche, V. E. Lyubovitskij, I. Schmidt, and A. Vega, Phys. Rev. D 82, 074022 (2010).

[12] J. Polchinski and M. J. Strassler, Phys. Rev. Lett. 88, 031601 (2002).

[13] D. Abbott et al. (JLAB t20 Collaboration), Eur. Phys. J. A 7, 421 (2000).

[14] H. R. Grigoryan and A. V. Radyushkin, Phys. Rev. D 76, 095007 (2007).

[15] C. Zhang et al., Phys. Rev. Lett. 107, 252501 (2011). 

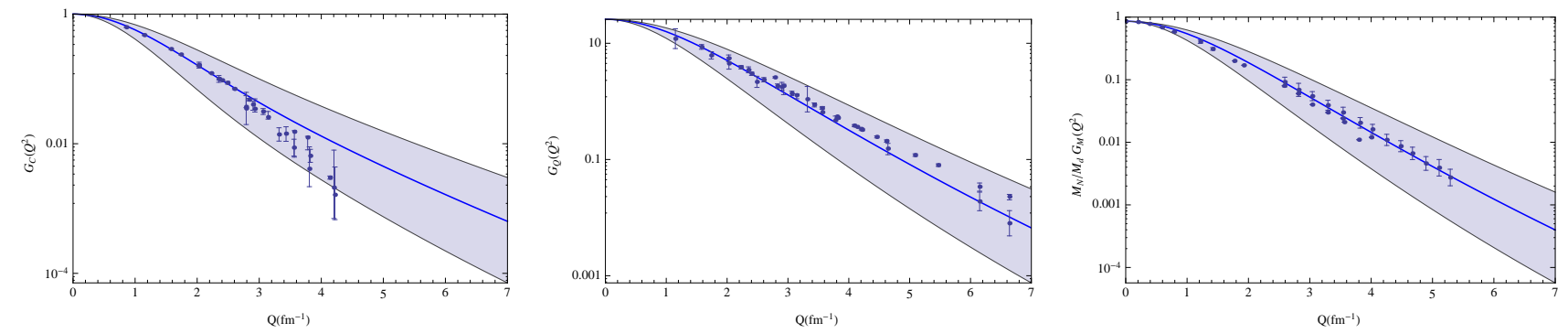

FIG. 1: Charge $G_{C}\left(Q^{2}\right)$, quadrupole $G_{Q}\left(Q^{2}\right)$ and magnetic $\left(m_{N} / m_{D}\right) G_{M}\left(Q^{2}\right)$ deuteron form factor.
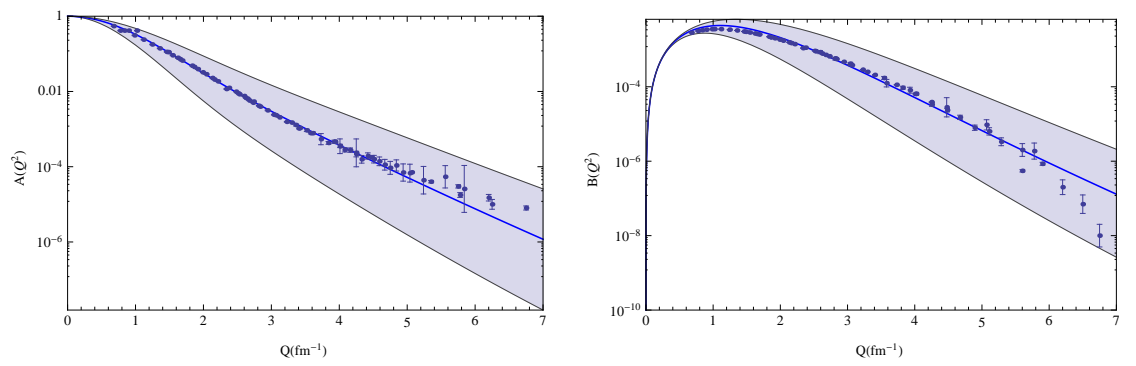

FIG. 2: Deuteron structure functions $A\left(Q^{2}\right)$ and $B\left(Q^{2}\right)$.
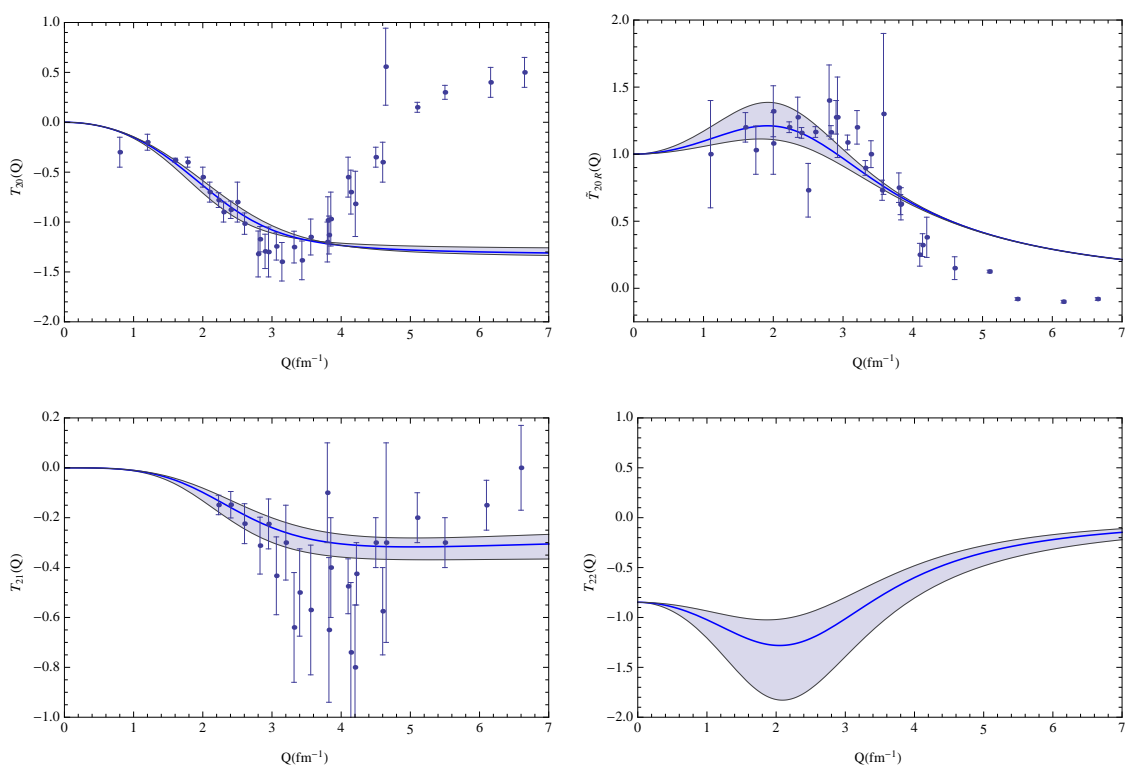

FIG. 3: Deuteron tensor-polarized structures $T_{20}\left(Q^{2}\right), \tilde{T}_{20 R}\left(Q^{2}\right), T_{21}\left(Q^{2}\right)$ and $T_{22}\left(Q^{2}\right)$. 

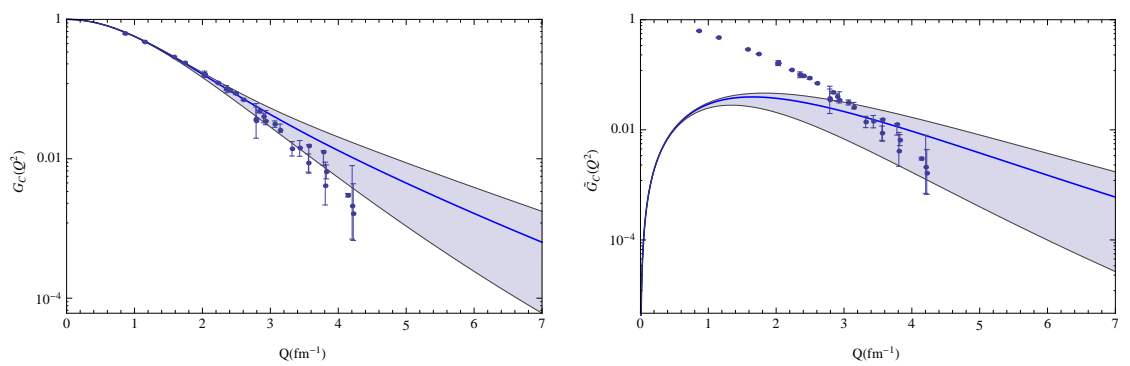

FIG. 4: Results for the charge form factor $G_{C}\left(Q^{2}\right)$ with the parameters in the range of $0 \leq \beta \leq 2$ (solid line corresponds to the $\beta=1.2$ ) and for the fixed values $\alpha_{2}=0.25, \alpha_{3}=1.1$ : exact result (left panel) and truncated result $\tilde{G}\left(Q^{2}\right)$ (right panel).
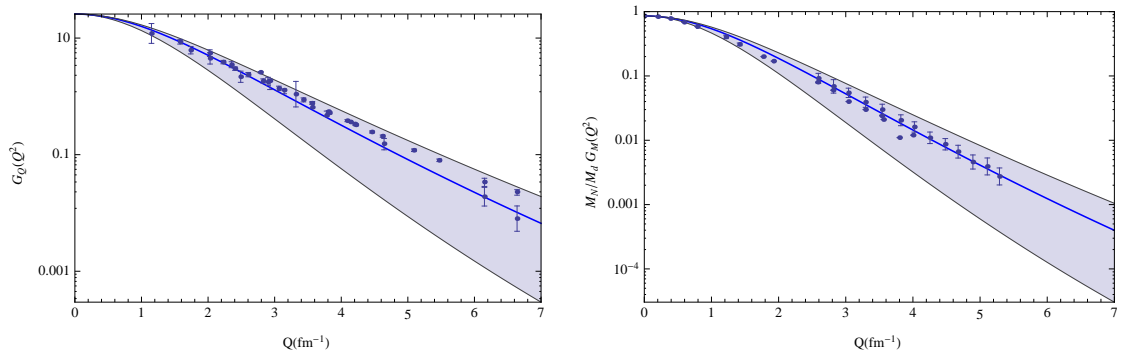

FIG. 5: Quadrupole $G_{Q}\left(Q^{2}\right)$ and magnetic $\left(m_{N} / m_{D}\right) G_{M}\left(Q^{2}\right)$ deuteron form factors for the parameters $0 \leq \beta \leq 2$ (solid line corresponds to the $\beta=1.2$ ) and $\alpha_{2}=0.25, \alpha_{3}=1.1$.
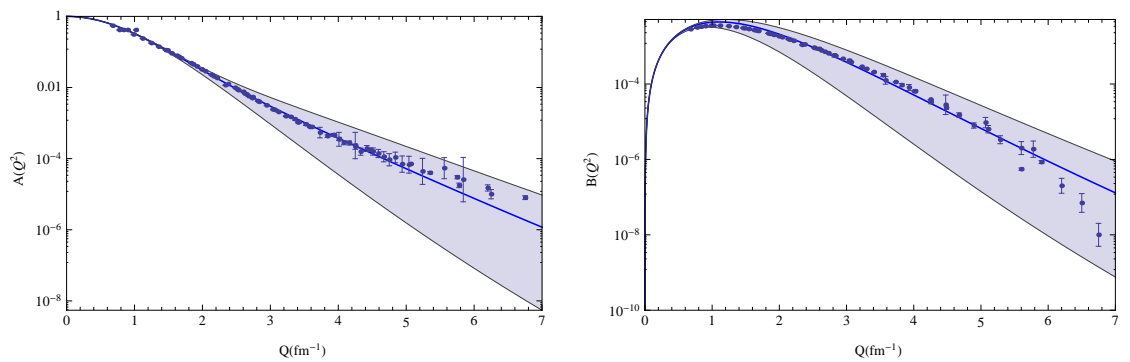

FIG. 6: Structure functions $A\left(Q^{2}\right)$ and $B\left(Q^{2}\right)$ for the parameters $0 \leq \beta \leq 2$ (solid line corresponds to the $\beta=1.2$ ) and $\alpha_{2}=0.25, \alpha_{3}=1.1$. 

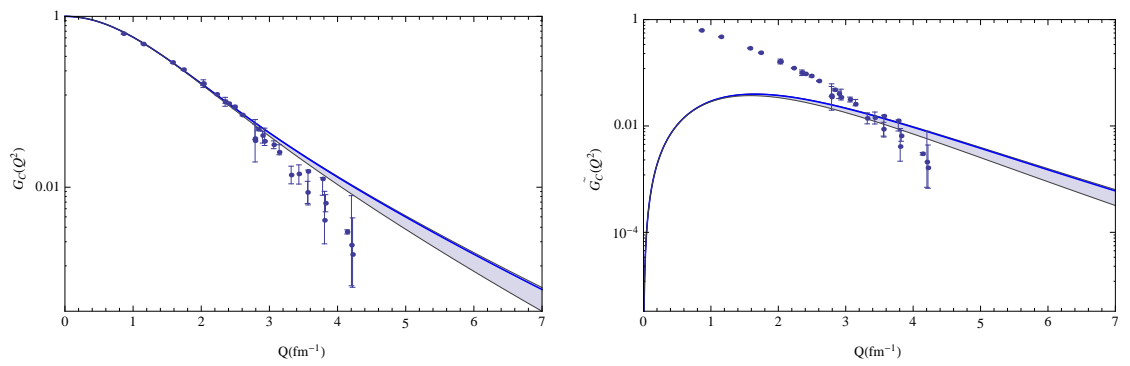

FIG. 7: Results for the charge form factor $G_{C}\left(Q^{2}\right)$ with the parameters $0 \leq \alpha_{2} \leq 1,1 \leq \alpha_{3} \leq 2$ (solid line corresponds to the $\alpha_{2}=0.25$ and $\alpha_{3}=1.1$ ) and $\beta=1.2$ : exact result (left panel) and truncated result $\tilde{G}_{C}\left(Q^{2}\right)$ (right panel).
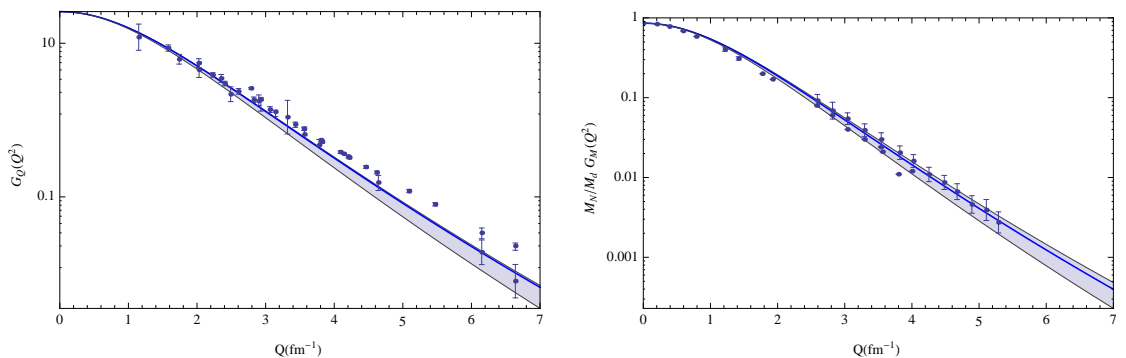

FIG. 8: Quadrupole $G_{Q}\left(Q^{2}\right)$ and magnetic $\left(m_{N} / m_{D}\right) G_{M}\left(Q^{2}\right)$ deuteron form factors with the parameters $0 \leq \alpha_{2} \leq 1$, $1 \leq \alpha_{3} \leq 2$ (solid line corresponds to the $\alpha_{2}=0.25$ and $\alpha_{3}=1.1$ ) and $\beta=1.2$.
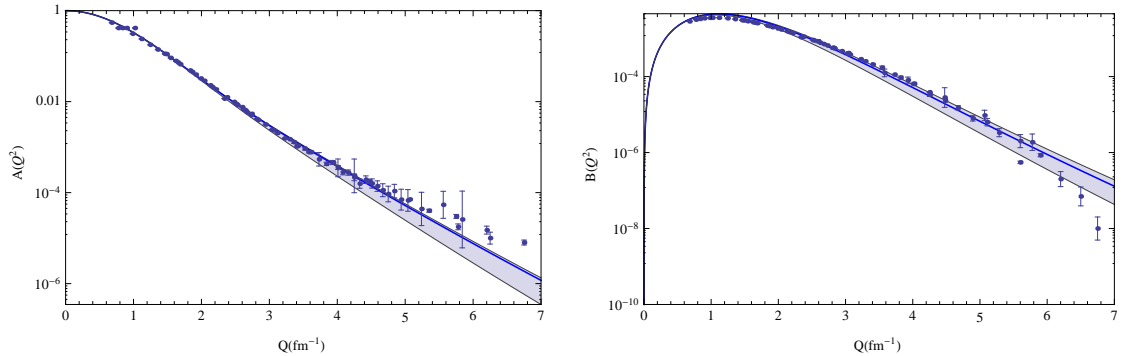

FIG. 9: Structure functions $A\left(Q^{2}\right)$ and $B\left(Q^{2}\right)$ with the parameters $0 \leq \alpha_{2} \leq 1,1 \leq \alpha_{3} \leq 2$ (solid line corresponds to the $\alpha_{2}=0.25$ and $\left.\alpha_{3}=1.1\right)$ and $\beta=1.2$. 